\title{
ANÁLISIS QUÍMICO Y MINERALÓGICO DE PINTURAS EN CERÁMICAS ARQUEOLÓGICAS DEL VALLE DE JEQUETEPEQUE Y SUS IMPLICANCIAS ARQUEOLÓGICAS
}

\author{
Nino Del-Solar-Velarde a \\ Rémy Chapoulie ${ }^{b}$ \\ Luis Jaime Castillo Butters ${ }^{c}$
}

\begin{abstract}
Resumen
En arqueología andina, el estudio de las formas y decoraciones de artefactos cerámicos es fundamental para la comprensión de la evolución de la cultura material de una o varias entidades colectivas. En el caso preciso de las decoraciones a base de pintura, su estudio se inicia con el registro adecuado a partir, por ejemplo, de observaciones macroscópicas y microscópicas en superficie o en sección. Estas observaciones se pueden complementar con la determinación de colores mediante el uso de uno o varios sistemas estandarizados de medida de color. Si bien estas prácticas se realizan con el fin de documentar, por ejemplo, la paleta de colores empleada por artesanos del pasado, es importante tener un conocimiento preciso sobre las materias primas y las recetas utilizadas en la producción de dichas pinturas; y es que la caracterización de unas y otras permitirá acceder, al menos de forma inicial, a la definición de las dinámicas socioeconómicas vinculadas a la explotación, uso y comercio de materiales asociados a la producción cerámica. Bajo estas premisas, y en el marco de este trabajo de investigación, se presentan los resultados de la caracterización quimica y mineralogica, mediante fluorescencia de rayos $X$ portátil ( $p X R F)$ y espectrometria Raman, de pinturas de un grupo de cerámicas mochica y cajamarca hallado en el sitio arqueológico de San José de Moro (valle de Jequetepeque, La Libertad, Perú).
\end{abstract}

Palabras clave: cerámica, pintura, mochica, cajamarca, arqueometría

\section{Abstract \\ CHEMICAL AND MINERALOGICAL ANALYSIS OF ANCIENT MOCHICA AND CAJAMARCA SLIPS FROM JEQUETEPEQUE VALLEY (PERU) AND THEIR ARCHAEOLOGICAL IMPLICATIONS}

In Andean archaeology, the study of vessel forms and slips is essential. The study of slips begins with macroscopic and microscopic observations on ceramic surfaces or sections and these observations can be complemented with the determination of colors applying one or more standardized systems of measure. These technical practices are executed in order to have a better knowledge about the palette de couleurs used by craftsmen in the past. Recently, with the new technologies applied to archaeology, it is possible to have a better knowledge of the recipes used in the elaboration of the slips, and thus, it is possible to contribute to the interpretation of social-economic dynamics regarding the ceramic production process. Following these premises, in this research we present the results of the chemical and mineralogical characterization, by means of portable X-ray fluorescence ( $p X R F)$ and Raman spectrometry, of ancient Mochica and Cajamarca slips (San José de Moro archaeological site, Jequetepeque Valley, La Libertad, Peru).

Keywords: ceramic, slip, Mochica, Cajamarca, archaeometry

${ }^{a}$ Ministerio de Cultura del Perú, Cusco

Correo electrónico: ninodelsolar@gmail.com

${ }^{\mathrm{b}}$ Université Bordeaux Montaigne

Correo electrónico: chapouli@u-bordeaux-montaigne.fr

$\simeq$ Pontificia Universidad Católica del Perú

Correo electrónico: 1castil@pucp.edu.pe 


\section{Introducción: ciencias experimentales, arqueología andina y cerámicas}

El estudio analítico de cerámicas arqueológicas - y las pinturas ${ }^{1}$ que las cubren- es prioritario en el ejercicio científico de la mayoría de programas y proyectos de investigación arqueológica en el Perú y en el área andina. El progreso de conocimientos obtenido gracias a la aplicación de un enfoque arqueométrico en el estudio del pasado cerámico peruano, sudamericano y latinoamericano es continuo e indiscutible, aunque, lamentablemente, tardío en relación al mismo fenómeno en el viejo mundo. En Europa, el uso de metodologías y la aplicación de técnicas de las ciencias experimentales en el estudio arqueológico fueron algo más tempranos y se consolidaron con la creación de laboratorios y revistas académicas especializadas entre los ańos 1950 y 1980.

Este progreso continuo e indiscutible se refleja, por ejemplo, en el conjunto de encuentros científicos, entorno a la aplicación de las ciencias experimentales en el estudio de bienes patrimoniales en países del área andina. Por citar dos casos, el primer Congreso Latinoamericano de Arqueometría, realizado en Argentina, data de 2007; o en el caso de Perú, el Congreso Nacional de Arqueología organizado por el Ministerio de Cultura, incluyó por primera vez, en 2014, una mesa entorno al estudio arqueométrico de vestigios arqueológicos nacionales.

Recientemente, nuevos encuentros científicos permiten el afianzamiento del uso de metodologías y técnicas de las ciencias experimentales en el estudio del pasado cerámico andino. Uno de estos eventos fue el simposio internacional «Avances en el análisis de cerámica y pigmentos», organizado por la Pontificia Universidad Católica del Perú en junio de 2018, y que constituyó un encuentro académico significativo, en el que, por primera vez, especialistas se reunieron con el objetivo de evaluar los últimos progresos, las perspectivas de trabajo y las pistas de investigación en torno a la arqueometría de cerámicas y de pigmentos en países andinos como Argentina, Chile, Ecuador y Perú. Justamente, en el marco de la difusión de las investigaciones que se expusieron en este simposio, se presentan los resultados de los análisis químicos (realizados in situ) y mineralógicos (realizados ex situ), de pinturas de cerámicas arqueológicas mochica, cajamarca costeño y cajamarca serrano, así como las implicancias que podrían tener dichos resultados en la lectura arqueológica y tecnológica de las sociedades que las produjeron. Hecha esta sucinta y parcial reseńa histórica que tiene como objetivo situarnos, de alguna forma, en un contexto temporal de la investigación arqueológica y analítica de cerámicas andinas, es necesario introducir el caso de estudio que nos compete. En ese sentido, partiremos de las informaciones concernientes a la localización y función del sitio arqueológico de donde provienen las cerámicas arqueológicas estudiadas: el sitio San José de Moro.

El sitio arqueológico San José de Moro (distrito de Chepén, provincia de Pacanga, departamento de La Libertad), es un yacimiento mochica excepcional, localizado en la parte baja del valle de Jequetepeque (Costa Norte del Perú, Fig. 1) que funcionó, primordialmente, como centro regional de actividades rituales, espacio de encuentro social y cementerio de elite (Castillo 2000, 2003, 2008, 2010, 2011, 2012). Una de las características que hacen de San José de Moro un sitio particular dentro del área mochica, es la convergencia y presencia concomitante, en contextos arqueológicos del período Mochica Tardío (c. s. VIII-IX d.C.) y período Transicional (c. s. IX-XI d.C.), de materiales arqueológicos de sociedades y grupos costeros y serranos como los «Mochica (que ocuparon [...] primordialmente el medio costero del norte del Perú) y [... los] Cajamarca (asociados a Huari, y cuyas principales comunidades ocuparon fundamentalmente la zona quechua entorno al valle de Cajamarca)» (Del-Solar-Velarde et al. 2017: 169).

El estudio sistemático de los vestigios arqueológicos que se hallan en este sitio ha sido desarrollado por el Programa Arqueológico San José de Moro, de la Pontificia Universidad Católica del Perú, en coordinación académica con instituciones como la National Geographic Society, la Universidad de Harvard, la Universidad de California Los Ángeles, entre otras. Este programa multidisciplinario de investigación, que tiene sus orígenes en la década de 1990, conserva como principal objetivo la comprensión de las dinámicas de ocupación en el sitio arqueológico y en la parte baja del valle 

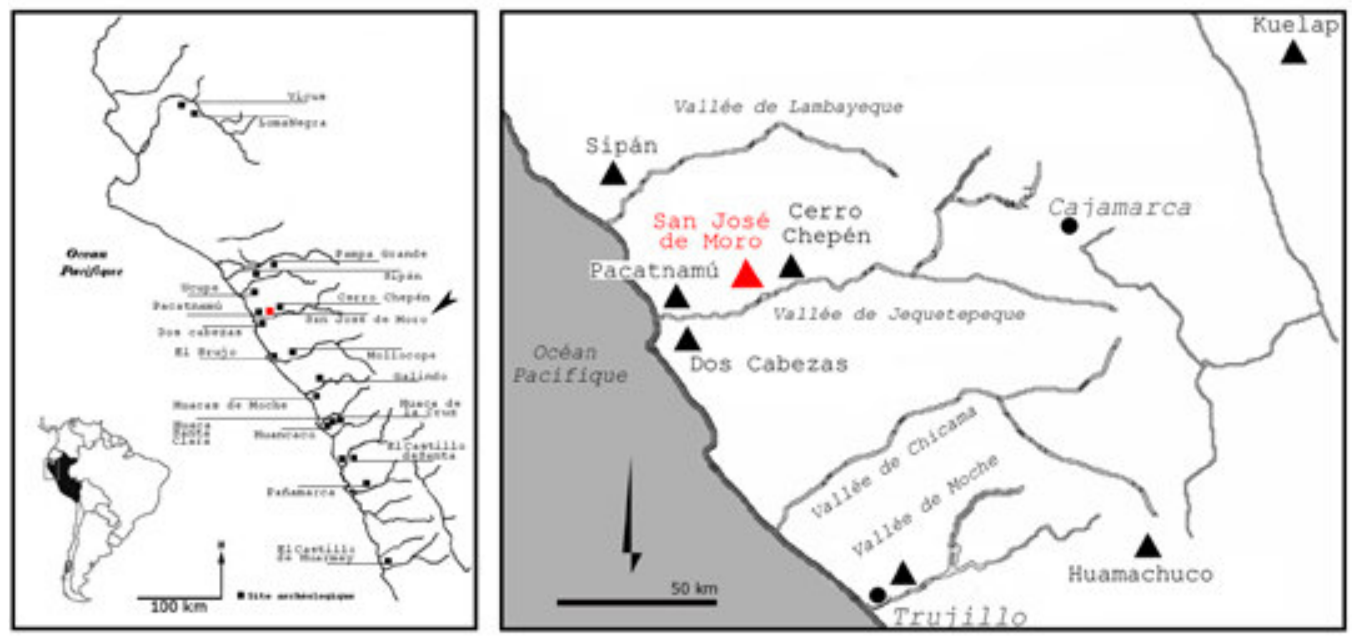

Figura 1. Localización del sitio arqueológico de San José de Moro en el valle de Jequetepeque, costa norte del Perú (Nino Del-Solar-Velarde, PASJM e IRAMAT-CRP2A).

de Jequetepeque. Ahora bien, el interés de caracterizar dichas dinámicas se basa, como se mencionó líneas arriba, en las particularidades del registro arqueológico que ofrece el sitio: en San José de Moro, desde las primeras intervenciones arqueológicas desarrolladas por Disselhoff (1958), se destacó la existencia convergente de vestigios de sociedades precolombinas que explotaron espacios geográficos diferentes; por ejemplo, restos materiales de los moche, sociedades que explotaron, principalmente, recursos del desierto subtropical (altitud aproximada: 0 a $1000 \mathrm{msnm}$ ) (Tosi 1960: 10, 14); y restos materiales de grupos cajamarca, quienes exploraron, esencialmente, materiales y recursos del bosque seco montano bajo (altitud aproximada: 2800 a $3400 \mathrm{msnm}$ ) (Tosi 1960: 10, 101-102).

Al igual que Bernuy y Bernal (2008: 67), creemos que San José de Moro constituye un sitio donde es posible estudiar de forma contextualizada, a partir de los vestigios materiales (mochica, cajamarca y huari), fenómenos de interacción regional y macrorregional. Ahora bien, el estudio de estos fenómenos no solo debe basarse en la identificación, registro y comparación de artefactos arqueológicos a partir de dos componentes mayores como son la forma y la decoración, componentes que varían rápidamente en el tiempo, sino que debe también basarse en el estudio y caracterización, por ejemplo, de los materiales con los que fueron elaborados o producidos, con el fin de establecer adecuadamente el carácter (social, económico y tecnológico) de dichas interacciones. En ese sentido, el registro, la documentación y el estudio analítico-comparativo de las características materiales de objetos arqueológicos (notablemente cerámicas y pigmentos) del valle de Jequetepeque, son labores que se han venido desarrollando al seno del Programa Arqueológico San José de Moro con el fin, además, de establecer estilos técnicos de producción en la zona de estudio. Estas labores se ejecutaron y ejecutan en coordinación académica con instituciones dedicadas a la investigación en ciencias experimentales aplicadas al estudio de materiales arqueológicos o del patrimonio cultural material, como por ejemplo, el Instituto de Investigación de Arqueomateriales y Centro de Investigación en Física Aplicada a la Arqueología (IRAMAT-CRP2A) de la Universidad Burdeos Montaigne (Rohfritsch 2006; Thiriet 2008; Del-Solar-Velarde 2011, 2015; Dollwetzel 2012; Chapoulie et al. 2018), la Facultad de Ciencias de Materiales de la Universidad Tecnológica de Chemnitz (Nickel et al. 2013), o el Instituto Federal Alemán para la Investigación y Test de Materiales (Dietrich et al. 2017, 2018).

Bajo todas estas premisas, estos análisis se realizaron en el marco de un estudio específico cuyo objetivo general fue identificar, establecer y documentar la naturaleza química y mineralógica de las materias primas empleadas en la producción de pinturas oscuras (de tonos rojo, marrón, marrón 
oscuro y/o negro) en cerámicas mochica, cajamarca costeño y cajamarca serrano, y utilizar esta información en la propuesta de hipótesis arqueológicas sobre la proveniencia y circulación de materiales.

Del mismo modo, los objetivos específicos de este estudio fueron tres. Primero, documentar y caracterizar químicamente, y por primera vez, pinturas rojas, marrones, marrones oscuro y/o negras en cerámicas mochica, cajamarca costeńo y cajamarca serrano, con un espectrómetro de fluorescencia de rayos X portátil (pXRF). Segundo, a partir de los resultados obtenidos del análisis químico o elemental, documentar y caracterizar mineralógicamente los principales compuestos en pinturas cerámicas, esto mediante espectrometría Raman. Y tercero, comparar los resultados con aquellos obtenidos por Chapdelaine et al. (1997), Rohfritsch $(2006,2010)$ y Thiriet (2008) quienes identificaron el empleo de óxidos de hierro (principalmente hematita y la espinela magnetita) en pinturas oscuras en cerámicas mochica y cajamarca costeño y serrano, mediante otras técnicas como, por ejemplo, microscopía electrónica de barrido acoplada a un espectrométro de rayos $\mathrm{X}$ en dispersión de energía (MEB-EDS)².

\section{Materiales y métodos: pinturas en cerámicas mochica y cajamarca y su estudio analítico}

En el marco de este estudio, se presenta el análisis de los resultados de composición elemental y mineralógica de 49 ( $\mathrm{n}=49)$ pinturas oscuras (de tonos rojo, marrón, marrón oscuro y/o negro) en fragmentos de cerámicas mochica, cerámicas cajamarca costeño y cerámicas cajamarca serrano (Tabla 1, Figs. 2 y 3), hallados en el marco de los trabajos de investigación del Programa Arqueológico San José de Moro de la Pontificia Universidad Católica del Perú, temporadas 2013 y $2014^{3}$.

Los análisis químicos y mineralógicos se han realizado con técnicas no invasivas o no destructivas, in situ, durante los trabajos de investigación en gabinete en el mismo sitio arqueológico, y ex situ, en el Instituto de Investigación de Arqueomateriales y Centro de Investigación en Física Aplicada a la Arqueología (IRAMAT-CRP2A) de la Universidad Burdeos Montaigne.

En lo que concierne el tratamiento de materiales previo a los análisis, es necesario mencionar que, para efecto de aminorar la contaminación de las muestras debido a la presencia adherida de restos del medio sedimentario sobre las mismas, los fragmentos de cerámica han sido lavados con agua y secados in situ. Posteriormente, fueron lavados con agua desmineralizada y secados en horno a $45^{\circ} \mathrm{C}$ durante tres días, en los laboratorios del Instituto de Investigación de Arqueomateriales de Burdeos.

In situ, se empleó un espectrómetro Bruker Tracer III-SD handheld, equipo de la Pontificia Universidad Católica del Perú. En caso se haya realizado más de un análisis en un mismo color o tono, se ha procedido a obtener una media y una desviación estándar por pintura. Se han estudiado un total $38(\mathrm{n}=38)$ pinturas de cerámicas mochica del período Tardío (c. s. VIII-IX d. C.) y ocho $(\mathrm{n}=8)$ pinturas de cerámicas cajamarca costeño y cajamarca serrano producidas durante el período Mochica Tardío y período Transicional (c. s. VIII-XI).

En lo que concierne a los parámetros analíticos, se ha priorizado la detección de una gama de 13 elementos químicos ( $\mathrm{K}$ al Mo) y se han medido los rayos $\mathrm{X}$ de fluorescencia, líneas espectrales $\mathrm{K}$ de cada elemento detectado. Posteriormente, se ha realizado el estudio comparado de las intensidades de los picos de las líneas de emisión Ka1. Cabe señalar que los rayos X fueron producidos por una fuente de Rodio, a una aceleración de $40 \mathrm{kV}$, y una intensidad de corriente de $30 \mu \mathrm{A}$. El tiempo de adquisición fue de $120 \mathrm{~s}$ por pintura. Para los análisis no se aplicó el vacío. El área de análisis fue de 3 milímetros por 4 milímetros aproximadamente; en tal sentido, fue imposible realizar análisis puntuales en todas las pinturas debido a la geometría de los fragmentos y el área total pintada. Para la explotación de los datos obtenidos se han empleado los programas S1PXRF (Bruker AXS Handheld Inc. V3.8.30) y Bruker Spectra Artax (v.7.4.0.0).

De modo similar, en lo que concierne a los análisis ex situ, estos se realizaron de forma complementaria sobre once $(n=11)$ pinturas con el fin de explorar la composición mineralógica de las mismas. Para tales efectos, se utilizó un equipo Renishaw Raman RM2000 asociado a un microscopio 
Tabla 1. Fragmentos y sus pinturas objeto de estudio. Leyenda: +: pintura analizada mediante pXRF; *: pintura analizada mediante espectrometría Raman; DPM: decoración pintada mochica; DPC: decoración pintada cajamarca.

\begin{tabular}{|c|c|c|c|c|c|}
\hline & Objeto & Parte & Clasificación & Fragmento y pintura & Código \\
\hline 1 & Botella o jarra & Cuerpo & Mochica (Tardío) & BDX16317 marrón * & DPM1 \\
\hline 2 & Botella o jarra & Cuerpo & Mochica (Tardío) & BDX16324 marrón * & DPM2 \\
\hline 3 & Botella o jarra & Cuerpo & Mochica (Tardío) & BDX16326 marrón ${ }^{+}$ & DPM3 \\
\hline 4 & Botella o jarra & Cuerpo & Mochica (Tardío) & BDX16327 marrón + & DPM4 \\
\hline 5 & Botella o jarra & Cuerpo & Mochica (Tardío) & BDX16328 marrón rojizo ${ }^{+}$ & DPM5 \\
\hline 6 & Botella o jarra & Cuerpo & Mochica (Tardío) & BDX16330 marrón oscuro $^{+}$ & DPM6 \\
\hline 7 & Botella & Pico & Mochica (Tardío) & BDX16331 marrón oscuro $^{+}$ & DPM7 \\
\hline 8 & Botella & Asa & Mochica (Tardío) & BDX16332 marrón oscuro $^{+}$ & DPM8 \\
\hline 9 & Botella o jarra & Cuerpo & Mochica (Tardío) & BDX16333 marrón oscuro + & DPM9 \\
\hline 10 & Botella & Asa & Mochica (Tardío) & BDX16342 marrón + & DPM10 \\
\hline 11 & Botella & Pico & Mochica (Tardío) & BDX16343 marrón + & DPM11 \\
\hline 12 & Botella & Asa & Mochica (Tardío) & BDX16345 marrón + & DPM12 \\
\hline 13 & Botella & Asa & Mochica (Tardío) & BDX16487 marrón oscuro +* & DPM13 \\
\hline 14 & Botella & Asa & Mochica (Tardío) & BDX17162 marrón ${ }^{+}$ & DPM14 \\
\hline 15 & Botella & Asa & Mochica (Tardío) & BDX17163 marrón $^{+}$ & DPM15 \\
\hline 16 & Botella & Asa & Mochica (Tardío) & 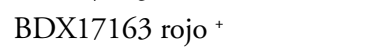 & DPM1C \\
\hline 17 & Botella & Asa & Mochica (Tardío) & BDX17166 marrón $^{+}$ & DPM17 \\
\hline 18 & Botella & Asa & Mochica (Tardío) & BDX17167 marrón + & DPM18 \\
\hline 19 & Botella o jarra & Cuerpo & Mochica (Tardío) & BDX17169 marrón +* & DPM19 \\
\hline 20 & Botella o jarra & Cuerpo & Mochica (Tardío) & BDX17170 marrón + & DPM20 \\
\hline 21 & Botella o jarra & Cuerpo & Mochica (Tardío) & BDX17172 marrón ${ }^{+}$ & DPM21 \\
\hline 22 & Botella o jarra & Cuerpo & Mochica (Tardío) & BDX17173 rojo + & DPM22 \\
\hline 23 & Botella o jarra & Cuerpo & Mochica (Tardío) & BDX17174 marrón $^{+}$ & DPM23 \\
\hline 24 & Botella o jarra & Cuerpo & Mochica (Tardío) & BDX17175 marrón ${ }^{+}$ & DPM24 \\
\hline 25 & Botella o jarra & Cuerpo & Mochica (Tardío) & BDX17176 marrón oscuro $^{+}$ & DPM25 \\
\hline 26 & Botella o jarra & Cuerpo & Mochica (Tardío) & BDX17178 marrón ${ }^{+}$ & DPM26 \\
\hline 27 & Botella o jarra & Cuerpo & Mochica (Tardío) & BDX17179 marrón ${ }^{+}$ & $\mathrm{DPM} 27$ \\
\hline 28 & Botella o jarra & Cuerpo & Mochica (Tardío) & BDX17180 marrón ${ }^{+}$ & DPM28 \\
\hline 29 & Botella o jarra & Cuerpo & Mochica (Tardío) (?) & BDX17182 marrón ${ }^{+}$ & DPM29 \\
\hline 30 & Jarra & Borde & Mochica (Tardío) & BDX16334 negro $^{+}$ & DPM30 \\
\hline 31 & Botella & Cuerpo & Mochica (Tardío) & BDX16336 negro + & DPM31 \\
\hline 32 & Botella & Cuerpo & Mochica (Tardío) & BDX16336 rojo + & DPM32 \\
\hline 33 & Vasija & Cuerpo & Mochica (Tardío) & BDX16338 negro $^{+}$ & DPM33 \\
\hline 34 & Vasija & Cuerpo & Mochica (Tardío) & 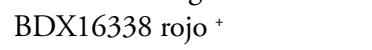 & DPM34 \\
\hline 35 & Botella & Cuerpo & Mochica (Tardío) & BDX16340 rojo ${ }^{+*}$ & DPM35 \\
\hline 36 & Jarra & Borde & Mochica (Tardío) & $\mathrm{BDX} 6488$ rojo $^{+}$ & DPM36 \\
\hline 37 & Botella & Asa & Mochica (Tardío) & BDX17164 negro $^{+}$ & DPM37 \\
\hline 38 & Botella & Asa & Mochica (Tardío) & $\mathrm{BDX} 17164$ rojo $^{+}$ & DPM38 \\
\hline 39 & Botella & Asa & Mochica (Tardío) & BDX17165 marrón +* & DPM39 \\
\hline 40 & Botella & Asa & Mochica (Tardío) & $\mathrm{BDX} 17165$ rojo $^{+*}$ & DPM40 \\
\hline 41 & Plato & Borde & Cajamarca costeño & BDX16556 rojo ${ }^{+*}$ & $\mathrm{DPC} 1$ \\
\hline 42 & Plato & Borde & Cajamarca costeño & BDX16556 marrón +* & DPC2 \\
\hline 43 & Plato & Borde & Cajamarca costeño & $\mathrm{BDX} 16560$ rojo $^{+}$ & DPC3 \\
\hline 44 & Plato & Borde & Cajamarca costeño & BDX16561 negro $^{+}$ & DPC4 \\
\hline 45 & Plato & Borde & Cajamarca serrano & $\mathrm{BDX} 6563 \mathrm{rojo}^{+}$ & DPC5 \\
\hline 46 & Plato & Borde & Cajamarca serrano & BDX16565 negro ${ }^{+*}$ & DPC6 \\
\hline 47 & Plato & Borde & Cajamarca serrano & $\mathrm{BDX} 6565$ rojo $^{+}$ & DPC7 \\
\hline 48 & Plato & Cuerpo & Cajamarca serrano & BDX16566 negro +* & DPC8 \\
\hline 49 & Plato & Borde & Cajamarca serrano & $\mathrm{BDX} 6567$ negro $^{+}$ & DPC9 \\
\hline
\end{tabular}



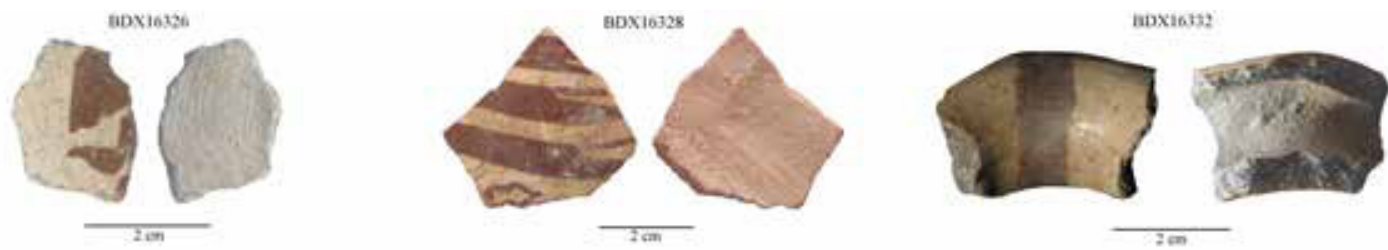

Boxisas?
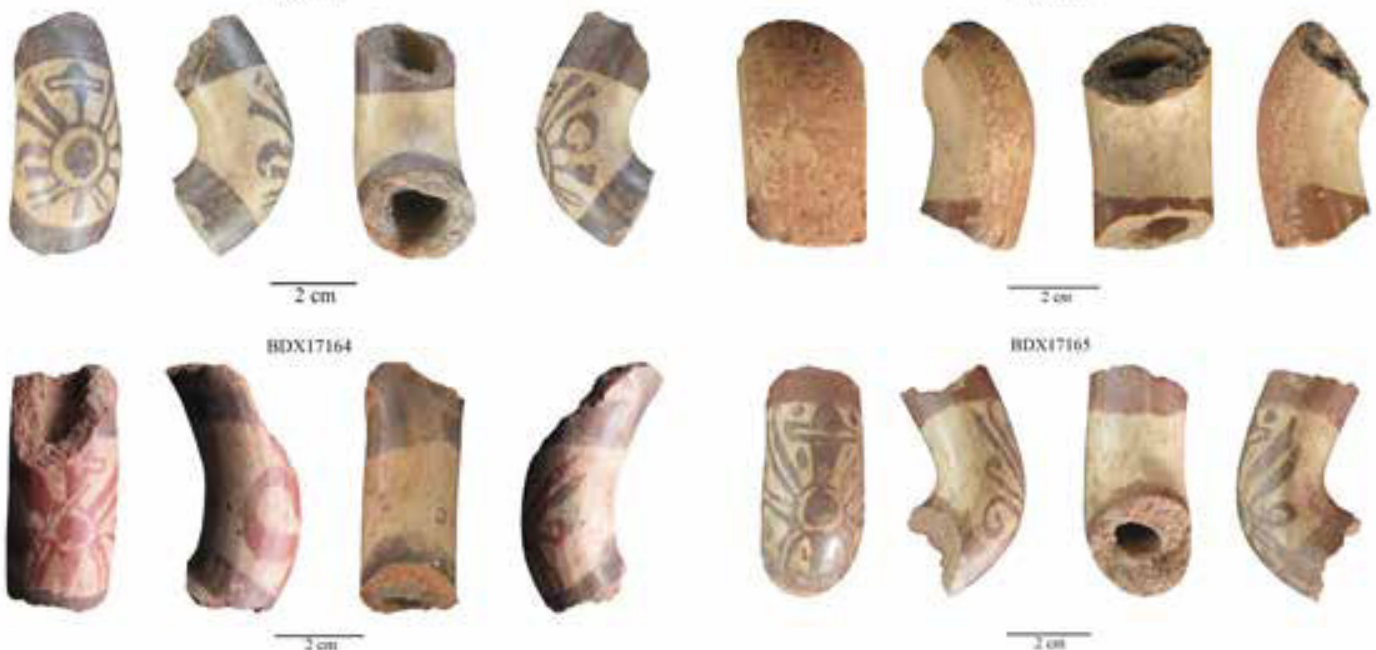

Figura 2. Fragmentos de cerámicas mochica (Nino Del-Solar-Velarde, PASJM e IRAMAT-CRP2A).
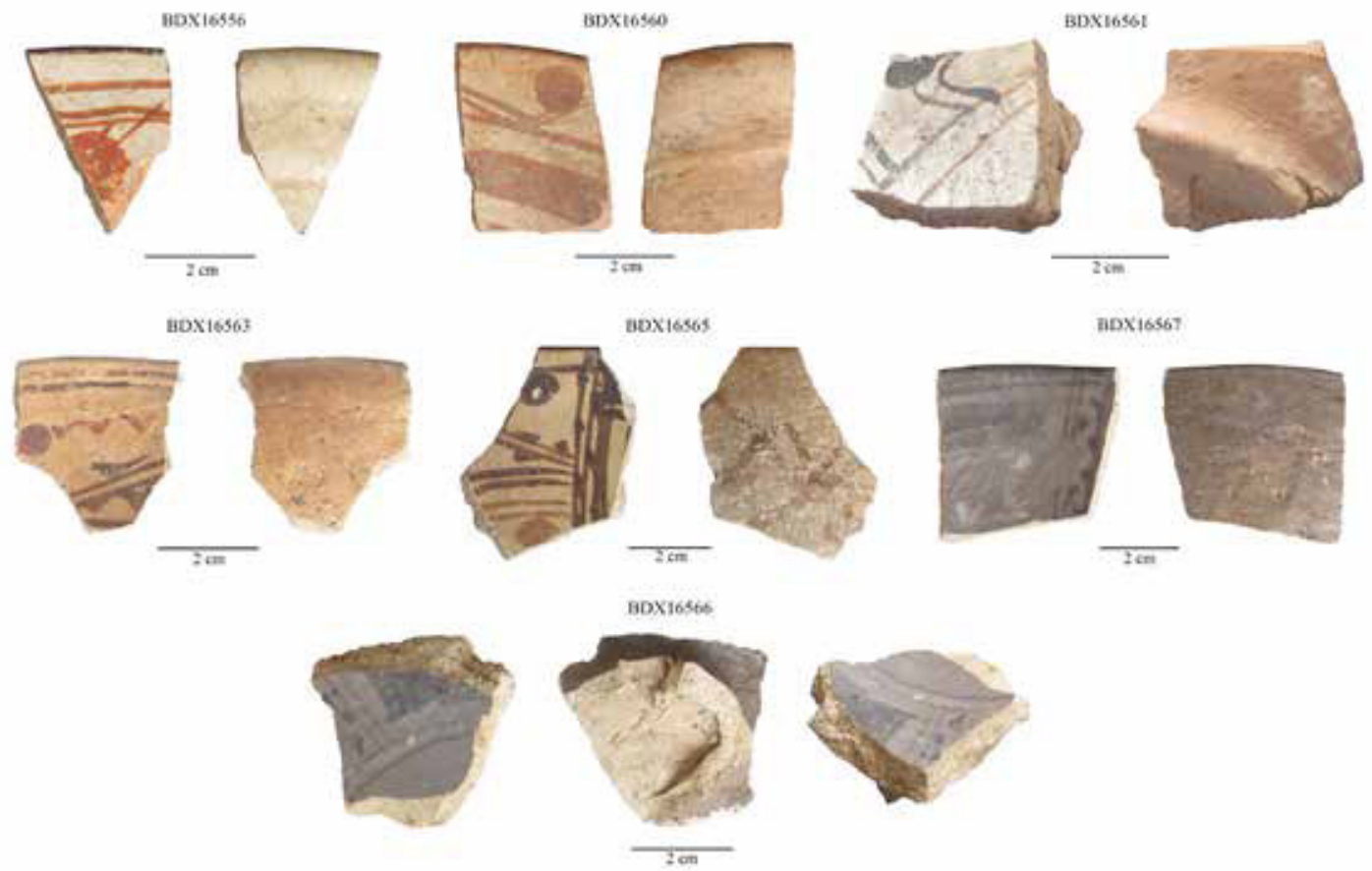

Figura 3. Fragmentos de cerámicas cajamarca (costeño y serrano) (Nino Del-Solar-Velarde, PASJM e IRAMAT-CRP2A). 
Leica DLLM (se trabajó a un aumento de X50, lo que permitió realizar análisis puntuales, de aproximadamente un micrómetro cuadrado). Si bien el equipo cuenta con tres láseres, solo se ha empleado el que posee una longitud de onda de $633 \mathrm{~nm}$, en modo continuous extended grating. Se han realizado de tres a cinco adquisiciones por pintura y cada adquisición tuvo una duración de 10 s. El programa de tratamiento de espectros fue WiRE (Windows Raman Environment v1.3.18) y RRUFF Project - CristalSleuth Application (v. 19 de mayo, 2008).

\section{Resultados: documentación y caracterización analíticas}

Producto de los análisis químicos por fluorescencia de rayos X portátil, destaca la confirmación, mediante una técnica analítica que por primera vez se utiliza en el estudio de estos materiales, de la existencia de pigmentos previamente establecidos; así como la identificación de nuevos cromóforos al seno de pinturas mochica y cajamarca.

En ese entender, cabe señalar que, en más del 75\% de los análisis, los espectros de fluorescencia de rayos $\mathrm{X}$ portátil de pinturas (rojo, marrón, marrón oscuro y/o negro) en cerámicas mochica y cajamarca se caracterizaron por poseer picos de Fe de fuerte intensidad. El hierro, dentro de soluciones coloidales ricas en silicio y aluminio, constituyó el principal elemento para la producción de pinturas empleadas en la producción cerámica de ambas sociedades. Tanto en medio costero y serrano, minerales ricos en hierro aparecen de forma común, por lo que las hipótesis sobre explotaciones locales pueden ser de primer orden.

Ahora bien, si el hierro es el elemento químico que posee la tendencia a ser el más representativo y abundante en las pinturas analizadas, la comparación de las intensidades de los picos de las líneas de emisión $\mathrm{K} \alpha 1$ ha sido eficaz, pues ha permitido establecer, por primera vez, otro elemento químico agente de color. Se trata del manganeso que, en algunas pinturas, presenta concentraciones elevadas (Fig. 4).

A este respecto, fue clave la información suministrada por las relaciones entre el manganeso y el hierro al seno de la mayoría de pinturas (rojo, marrón, marrón oscuro y/o negro). Estas relaciones se hallan comúnmente entre 0.01 y 0.04 , con una media de 0.02 . A través de comparaciones, se ha podido observar que en algunos casos particulares, sobre todo en pinturas que presentan los tonos más oscuros y que se dirigen hacia el negro, la relación $\mathrm{MnK} \alpha 1 / \mathrm{FeK} \alpha 1$ fue igual o superior a 0.06 (Fig. 5). Se trata de las pinturas DPM8, DPM13, DPM18, DPM31, DMP33, DPM37, DMP39, DPC6, DPC8 y DPC9. Estas son pinturas mochica y cajamarca serrano que poseen cromóforos inorgánicos compuestos fundamentalmente por óxidos de manganeso o minerales de hierro y manganeso. Es decir, para su elaboración se emplearon arcillas a las que se añadieron minerales de manganeso y/o minerales de manganeso y hierro. En el caso de las pinturas mochica (DPM8, DPM13, DPM18, DPM31, DMP33, DPM37 y DMP39), es posible sugerir que en su fabricación se emplearon arcillas ferruginosas naturales a las que se añadieron minerales de manganeso.

Por otra parte, los análisis mineralógicos mediante espectrometría Raman han tomado tiempo y han sido bastante complejos, debido a que los materiales analizados emitían, en repetidas ocasiones, espectros con ruido elevado. A pesar de la dificultad operativa en la aplicación de la técnica, los análisis han permitido conocer la composición mineralógica de las pinturas cerámicas. Si bien los resultados por espectrometría Raman han corroborado parte de lo señalado por Rohfritsch (2006, 2010) y Thiriet (2008), pues si bien se ha observado la presencia de hematita $\left(\mathrm{Fe}_{2} \mathrm{O}_{3}\right)$ en la mayoría de pinturas marrón o rojo de cerámicas mochica y cajamarca, halladas en San José de Moro (por ejemplo DPM1, DPM19 o DPC1), nuestro análisis ha permitido identificar, además de hematita y magnetita, la presencia de minerales del grupo de la espinela como la jacobsita $\left(\mathrm{MnFe}_{2} \mathrm{O}_{4}\right)$ en algunas pinturas oscuras mochica, y la hausmanita $\left(\mathrm{Mn}_{3} \mathrm{O}_{4}\right)$ en pinturas negras de cerámicas cajamarca serrano (Fig. 6). 

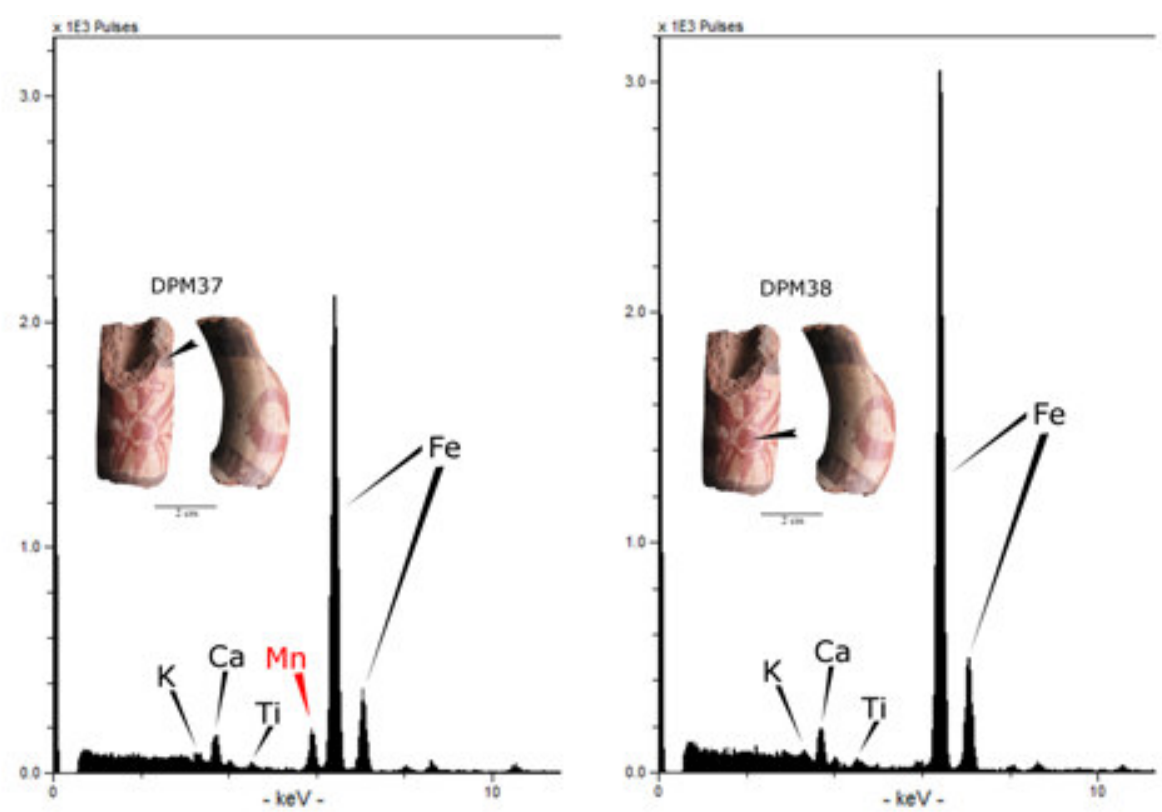

Figura 4. Espectros de fluorescencia de rayos X obtenidos del análisis de pinturas en el fragmento BDX17164 (Nino DelSolar-Velarde, PASJM e IRAMAT-CRP2A).

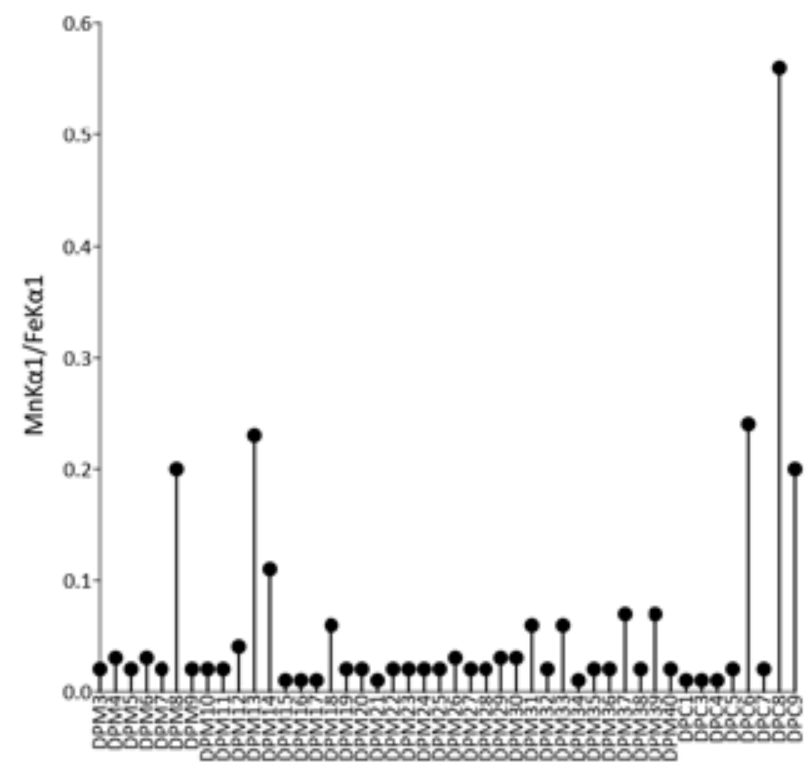

Figura 5. Relaciones de las intensidades MnKal y FeKa1, ambas obtenidas del análisis mediante $\mathrm{pXRF}$ de pinturas (en tonos rojo, marrón, marrón oscuro y/o negro) en cerámicas mochica y cajamarca (Nino Del-SolarVelarde, PASJM e IRAMAT-CRP2A).

Probablemente, entre las posibles razones por las que anteriormente no se pudo identificar o detectar dichas estructuras cristalinas, creemos pertinente citar al menos dos: 1) la confusión con la presencia de magnetita (estructura que genera macroscópicamente tonalidades similares y cuyo pico mayor en su espectro Raman se encuentra en 673 Raman Shift $\left(\mathrm{cm}^{-1}\right)$; y 2) la falta de análisis químicos directos complementarios que habrían permitido ver otros elementos químicos cromógenos complementarios al hierro. 

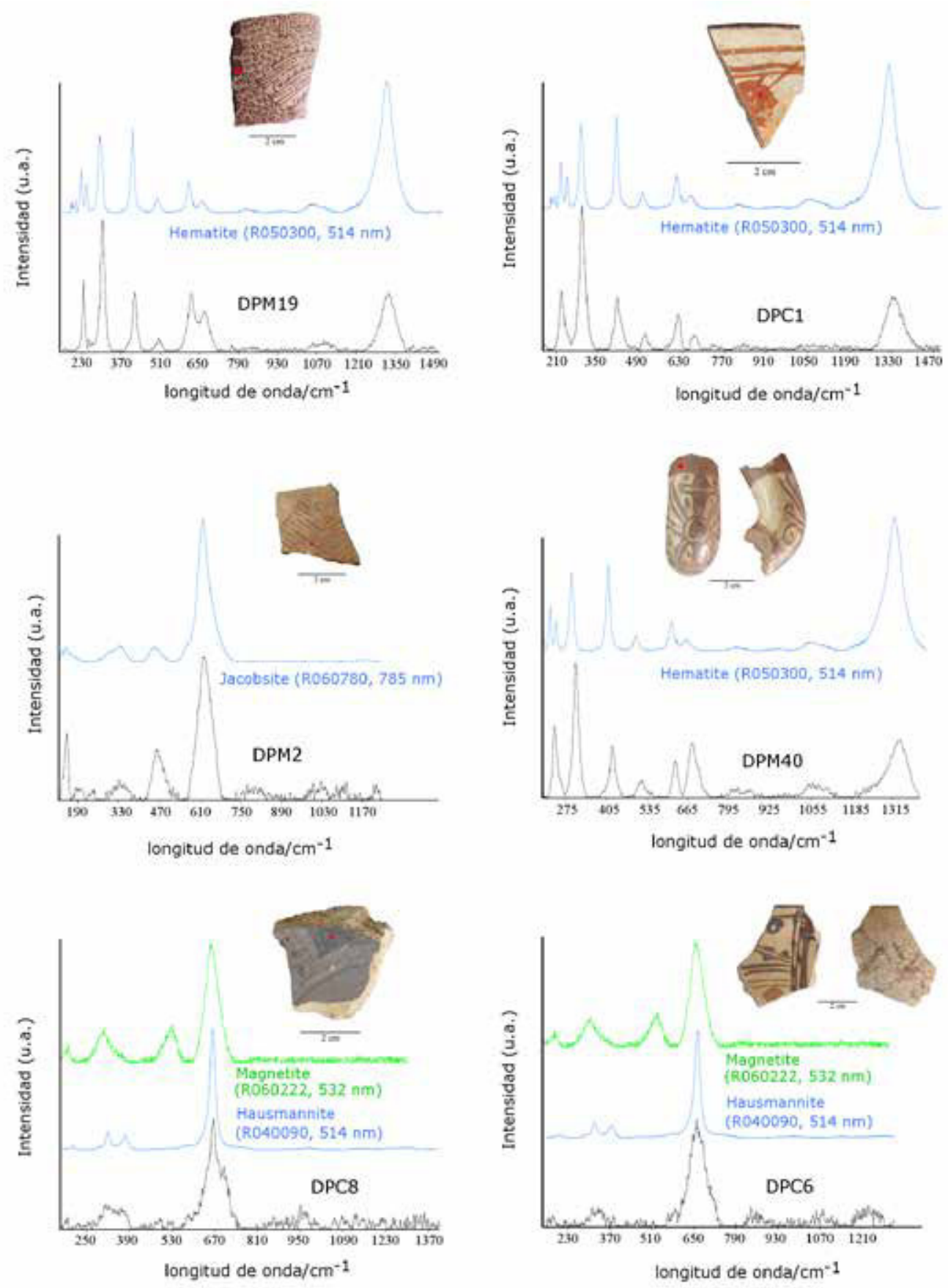

Figura 6. Espectros Raman obtenidos sobre pinturas cerámicas mochica y cajamarca (Nino Del-Solar-Velarde, PASJM e IRAMAT-CRP $2 A$ ). 


\section{Discusión: implicancias arqueológicas y pistas de investigación}

Desde un punto de vista técnico, el análisis químico y mineralógico, in situ y ex situ, de pinturas cerámicas mochica y cajamarca ha sido fructífero debido a su complementariedad al momento de explicar los principales cromóforos empleados en sus elaboraciones.

Desde un punto de vista arqueológico, aún son pocos y restringidos los estudios analíticos en los que se ha caracterizado los sistemas técnicos mochica, cajamarca costeńo y cajamarca serrano. Más aún, si estos han tratado de ser caracterizados, no ha existido, en la mayoría de casos, estudios secuenciales y por períodos, lo que dificulta la observación de cambios en patrones tecnológicos o de producción a lo largo del tiempo y en áreas definidas. Por ejemplo, a futuro, es necesaria la ejecución de un estudio exclusivo de los sistemas técnicos de los períodos Mochica Temprano y Mochica Medio, o estudios particulares para la comprensión tecnológica de las producciones cerámicas de cada una de las subfases del período Cajamarca Medio, en uno o varios de sus sitios de ocupación.

En el caso de la pintura roja, marrón, marrón oscuro o negro en vasijas mochica, las investigaciones arqueométricas de años pasados propusieron, en más de una vez, el uso de minerales de hierro para su elaboración. Nuestro estudio permite señalar que San José de Moro y su cerámica mochica, no son la excepción de lo que puede ser una norma técnica, ya que en la mayoría de casos los principales cromóforos inorgánicos empleados son los óxidos de hierro. En otras palabras, la producción de estas pinturas presenta un lenguaje tecnológico común con relación a pinturas en cerámicas de otro sitio moche como, por ejemplo, las Huacas del Sol y de la Luna.

Ahora bien, a pesar de lo señalado, el hallazgo e identificación del uso de minerales de manganeso en algunas pinturas (en marrón, marrón oscuro o negro) en cerámicas mochica del período Tardío, permite, sin duda alguna, proponer o formular cuestiones entorno a la importación de formas y motivos (por ejemplo, formas y diseños huari) acompañada del préstamo de algunas soluciones tecnológicas y materiales, sobre todo en un momento y período en el que no solo se importan a la costa objetos de Ayacucho y de Cajamarca sino que se comienzan a producir, dentro de los talleres mochica, artefactos polícromos, híbridos, con motivos huari (chakipampa) y moche. En este punto, es necesario presentar dos nociones que sustentan esta propuesta: primero, aún no se conocen de forma precisa fuentes de minerales de manganeso en la costa norte moche; y segundo, el empleo de minerales de manganeso para la pintar objetos de tierra (cocida o no) en los sistemas técnicos del Perú precolombino fue conocido, practicado y dominado ${ }^{4}$. Estos minerales o materias primas han sido identificados en pinturas de artefactos asociados, principalmente, al fenómeno huari, así como en pinturas de objetos y arquitectura de otros períodos y espacios culturales, por ejemplo: Donnan (1972) los identificó en pinturas murales mochica-huari del sitio Huaca Facho, en el valle de Lambayeque; Bonavia (1985) propuso su uso para la elaboración de pinturas murales del sitio La Mayanga, en el valle de La Leche; Proulx (2000) estableció su empleo en la preparación de pinturas en cerámicas nasca; Swann et al. (1999) planteó, a través de una evaluación comparativa, su presencia en pinturas oscuras sobre cerámicas shipibo/cumancaya, una tradición cerámica de sociedades asentadas en torno al río Ucayali; Del-Solar-Velarde (2011) los identificó en pinturas negras de cerámicas huari de la zona de Ayacucho; Del-Solar-Velarde (2014) y Sardos (2017) establecieron su uso en pinturas murales del sitio de San José de Moro; Druc (2014) estableció su empleo, durante el Período Formativo de Puémape, para producir el negro de una botella cupisnique; Druc et al. (2015 y en este volumen) determinaron la existencia de pigmentos negros a base de manganeso en cerámicas Rojo Grafitado; Del-Solar-Velarde (2018a, 2018b) identificó su uso en la producción de pinturas violetas y negras de cerámicas huari e inca de la región de Cusco; y Wright (comunicación personal) los halló en pinturas murales negras del sitio inca de Tambo Colorado (provincia de Pisco).

En el caso de las producciones cajamarca, se ha corroborado el uso de minerales ricos en hierro en pinturas rojas de cerámicas Cajamarca costeño, y por primera vez se ha establecido la presencia 
de cromóforos inorgánicos a base de manganeso en pinturas de cerámicas cajamarca serrano. Es fundamental señalar que recientes estudios ejecutados por Druc (2011) y Druc et al. (2013) han identificado la presencia de fuente(s) y el uso de minerales de manganeso por parte de artesanos tradicionales en la zona de Cajamarca para decorar cerámicas modernas o réplicas, lo que apoyaría e iría de la mano a nuestra identificación en pinturas de cerámicas arqueológicas producidas y provenientes de esta zona y región del Perú.

Si la arqueología ha demostrado que la sociedad mochica de San José de Moro importó objetos polícromos huari y objetos cajamarca durante el período Mochica Tardío, no es desatinado ni incoherente pensar que los talleres de cerámica moche de la zona pudieron, igualmente, haber importado e incorporado materias primas empleadas por artesanos de estas sociedades con el fin de experimentar y obtener una mayor gama de tonalidades oscuras para pintar sus cerámicas de prestigio. ¿Acaso estas materias primas provenían de la zona vecina de Cajamarca? A este respecto, como se mencionó líneas arriba, recientes estudios han identificado una mina de manganeso en las cercanías de dicha zona (Druc 2011; Druc et al. 2013). En un momento en que los mochica dejan de ser tan herméticos, es posible que la bicromía clásica de su cerámica decayera en alguna medida, que la paleta de colores de sus artesanos se ampliara, y que los materiales de producción $-\mathrm{o}$ los insumos- se diversificaran debido a la necesidad sociopolítica de producir vasijas polícromas e híbridas, mochica-huari. Bajo la necesidad de producir objetos de prestigio polícromos y en un contexto de fuerte intercambio social con poblaciones serranas y quechuas, la hipótesis del flujo de tecnologías y materias primas aportadas por grupos vecinos y contemporáneos, como los cajamarca, es de primer orden y debe tenerse en cuenta en futuras investigaciones arqueológicas sobre los mochicas del valle de Jequetepeque.

Para finalizar, se esperan nuevas investigaciones que impliquen exclusivamente, por ejemplo, la identificación arqueológica de posibles fuentes de explotación de pigmentos minerales o la puesta en marcha de estudios isotópicos comparativos con materias primas del valle de Jequetepeque y del valle de Cajamarca. Entre tanto, los resultados de este trabajo permiten: primero, afirmar la importancia de la ejecución de estudios analíticos de materiales arqueológicos, como son las pinturas que cubrían las cerámicas, con equipos portátiles in situ. Es evidente que estas herramientas brindan resultados que, de un punto de vista cualitativo y semicuantitativo, son útiles y explotables siempre y cuando exista entrenamiento y dominio en su uso y en el tratamiento de la información obtenida; segundo, subrayar la necesidad de realizar investigaciones complementarias mediante el uso de más de una técnica analítica en la identificación de pigmentos precolombinos; y, tercero, proponer nuevas pistas de trabajo de investigación arqueológica para la Costa Norte, así como para el área andina, donde el estudio de las tecnologías y del comercio de materias primas empleadas para producir decoración, mejorar la impermeabilidad o comunicar ideas a través de motivos pintados sobre la superficie de los artefactos, se desarrolla cada vez de forma más robusta y apoyada en nuevas tecnologías y paradigmas.

\section{Agradecimientos}

Deseamos agradecer al Ministerio de Cultura del Perú por los permisos otorgados para la ejecución de los trabajos de investigación arqueológica en el sitio de San José de Moro. Agradecemos igualmente a la Universidad Burdeos Montaigne, al CNRS y el programa IdEx de Bordeaux (Initiative d'excellence de l'université de Bordeaux) por el soporte académico, técnico y financiero. Agradecemos a los editores por la lectura y las sugerencias realizadas sobre el manuscrito original. Finalmente, es preciso señalar que esta investigación ha beneficiado de una ayuda del Estado francés mediante la Agencia Nacional de la Investigación en el marco del programa de inversión Programme investissements d'avenir ANR-10-LABX-52/Laboratoire des sciences archéologiques de Bordeaux. 


\section{Notas}

1 Al inicio de la investigación, se había contemplado el uso general de los términos y la categoría 'decoración pintada' para referirnos a los motivos (por ejemplo: líneas rectas, cruces, círculos, espirales, seres antropomorfos, etc.) que se hallan pintados — en varios colores y tonalidades- directamente o sobre los engobes que cubren las cerámicas arqueológicas. Lamentablemente, al final de la investigación se terminó empleando estos términos y categoría de forma restringida, ya que pueden ser objeto de discusión: a pesar de que la categoría «decoración» es amplia y comúnmente empleada en la praxis arqueológica, por ejemplo, para la descripción, documentación y estudio de elementos, formas, motivos, diseńos u otros que se disponen o aparecen sobre la superficie interna o externa de un objeto - como una vasija cerámica-, es necesario mencionar que los fines de dichos elementos, formas o motivos pudieron no haber sido, original y estrictamente, decorativos. Según la Real Academia Española, se entiende por decoración al «conjunto de elementos que decoran... adornan...» o el "Arte que estudia la combinación de elementos ornamentales...» (RAE 2018). Todos los motivos y/o diseños que se pintaron directamente sobre cerámicas arqueológicas o sobre los engobes de cerámicas arqueológicas en el área andina no tuvieron, necesaria y primariamente, un rol decorativo, de adorno, de ornamentación o de embellecimiento, tal como se entienden estos conceptos en la actualidad. Estos pudieron haber tenido como rol primario el rol de comunicación de información, de ideas específicas vinculadas al sistema de creencias o de valores étnicos; el rol identitario o hasta el rol de legitimación étnica o grupal a través de la producción de bienes materiales con características específicas de forma y de diseño. Por tal motivo, si bien en la Tabla 1 se emplean los acrónimos DPM o DPC, por decoración pintada mochica o decoración pintada cajamarca, entenderíamos recomendable emplear conceptos más específicos y de menor ambigüedad como pintura en vez de «decoración pintada», y ampliar y enriquecer la discusión sobre la finalidad primaria de los materiales que cubrían artefactos cerámicos del Perú precolombino (por ejemplo, es posible que en algunas entidades colectivas de los Andes, los engobes no tuviesen necesaria y generalmente roles decorativos o informativos primarios, sino roles primarios tecnológicos al permitir que las superficies cerámicas, naturalmente porosas, se impermeabilicen de manera eficaz).

2 Bajo la premisa de que los objetivos de esta investigación incluyen documentar la composición química y mineralógica de las pinturas en cerámicas del sitio arqueológico San José de Moro, es necesario mencionar que ciertas características materiales de estas pinturas han podido desaparecer a lo largo del tiempo (a corto, mediano o largo plazo) debido, por ejemplo, a los efectos directos de la quema cerámica a temperaturas que sobrepasaron $\operatorname{los} 700{ }^{\circ} \mathrm{C}$, o a procesos de interacción entre material arqueológico y medio sedimentario. En tal sentido, documentar dichas características (por ejemplo, el uso de mínimas cantidades de materiales orgánicos en la preparación de las soluciones y pinturas) es cuestionable con las técnicas utilizadas, los parámetros analíticos establecidos y en los soportes (cerámicas) aquí estudiados.

3 Originalmente, este trabajo específico forma parte de una investigación más amplia desarrollada por Del-Solar-Velarde (2015). Este ha sido revisado y corregido. En ese sentido, la versión actual del artículo fue presentada en el evento simposio internacional «Avances en el análisis de cerámica y pigmentos» que se llevó a cabo en la Pontificia Universidad Católica del Perú, del 11 al 13 de junio de 2018.

${ }^{4}$ En el Perú precolombino, y precisamente en la Costa Norte del Perú, se han identificado otras soluciones técnicas para pintar con negro objetos de tierra (cocida o no). Una de esas soluciones engloba el uso de pigmentos a base de carbón o grafito. Por ejemplo, Wright (2007: 370) estableció el uso de pigmentos negros a base de carbón de madera para pinturas murales negras mochica estudiadas en el marco de su tesis doctoral, o a base de grafito más calcosiderita para pinturas murales negras de la sociedad lambayeque. 


\section{Referencias}

\section{Bernuy, K. y V. Bernal}

2008 La tradición Cajamarca en San José de Moro: una evidencia de interacción interregional durante el Horizonte Medio, en: L. J. Castillo, H. Bernier, G. Lockard y J. Rucabado (eds.), Arqueología mochica: nuevos enfoques. Actas del primer Congreso internacional de jóvenes investigadores de la cultura mochica (4-5 agosto 2004), 67-80, Pontificia Universidad Católica del Perú/Instituto francés de estudios andinos, UMIFRE 17-CNRS, Lima.

Bonavia, D.

1985 Mural painting in ancient Peru, Indiana University Press, Bloomington.

Castillo, L. J.

2000 La presencia Wari en San José de Moro, Boletín de Arqueología PUCP 4, 143-179.

2003 Los últimos mochicas en Jequetepeque, en S. Uceda y E. Mujica (eds.), Moche: hacia el final del milenio. Actas del segundo coloquio sobre la cultura moche (1-7 agosto 1999), vol. 2, 65-123, Pontificia Universidad Católica del Perú/Universidad Nacional de Trujillo, Lima.

2008 Prácticas funerarias de élite en San José de Moro, en: K. Makowski (ed.), Los Señores de los reinos de la luna, 288-293, Banco de Crédito del Perú, Lima.

2010 Moche politics in the Jequetepeque valley. A case for political opportunism, en J. Quilter y L. J. Castillo (eds.), New perspectives on the Moche political organization, 83-109, Dumbarton Oaks Research Library and Collection, Washington, D.C.

2011 San José de Moro y la arqueología del valle de Jequetepeque, Fondo Editorial PUCP, Lima.

2012 San José de Moro y el fin de los mochicas en el valle de Jequetepeque, Costa Norte del Perú, tesis de doctorado, Departamento de Antropología, Universidad de California Los Angeles, Los Angeles.

Chapdelaine, C., R. Mineau y S. Uceda

1997 Étude des pigments de la poterie cérémonielle Moche à l'aide d'un Microscope Électronique à Balayage, Bulletin de l'Institut Français d'Études Andines 26 (2), 229-245.

\section{Chapoulie, R., M. Sepúlveda, N. Del-Solar-Velarde y V. Wright}

2018 Arqueometría: estudios analiticos de materiales arqueológicos, Ediciones Institut Francais d'Études Andines, Université Bordeaux Montaigne y Universidad de Tarapacá, tomo 351, Colección Travaux de l'Institut Francais d'Études Andines, Lima.

Del-Solar-Velarde, N.

2011 La caractérisation archéométrique des céramiques précolombiennes du Pérou: une synthèse historique et méthodologique, Mémoire de Master 1, Université Michel-de-Montaigne Bordeaux 3, Burdeos.

2014 Caracterización elemental cualitativa y semicuantitativa por fluorescencia de rayos X portable de cerámicas, pigmentos y pinturas murales del sitio arqueológico San José de Moro, Perú, informe del Programa Arqueológico San José de Moro, Temporada 2014, 228-257.

2015 Contribution des sciences archéologiques à la connaissance des choix techniques Mochica et Cajamarca: étude des matériaux céramiques du site de San José de Moro (VIIIe-Xe s. apr. J.-C., Pérou), tesis de doctorado, Universidad Bordeaux Montaigne, Burdeos.

2018a Informe de análisis n ${ }^{\circ}$ 051-2018-NVDSV-DFQ-CCIA-AFPA-SDDPCDPC-DDC-CUS/MC del 13 de diciembre de 2018, propiedad de la Dirección Desconcentrada de Cultura de Cusco (análisis químico de cerámicas huari e inca del complejo arqueológico de Pikillaqta), Dirección Desconcentrada de Cultura de Cusco, Ministerio de Cultura del Perú, Cusco.

2018b Informe de análisis nº 054-2018-NVDSV-DFQ-CCIA-AFPA-SDDPCDPC-DDC-CUS/MC del 31 de diciembre 2018, propiedad de la Dirección Desconcentrada de Cultura de Cusco (análisis químico de cerámicas inca del sitio arqueológico de Kullupata), Dirección Desconcentrada de Cultura de Cusco, Ministerio de Cultura del Perú, Cusco.

\section{Del-Solar-Velarde, N., R. Chapoulie y L. J. Castillo}

2017 Caracterización geoquímica de pastas cerámicas mochica y cajamarca mediante microscopía electrónica de barrido acoplada a un espectrómetro de rayos X en dispersión de energía, Actas del Segundo Congreso Nacional de Arqueología, 169-176, Ministerio de Cultura del Perú, Lima. 
Dietrich, D., T. Mehner, N. Del-Solar-Velarde, D. Nickel, T. Lampke, R. Chapoulie y L. J. Castillo

2017 Archaeometric case studies on decorations of pre-Columbian pottery using EDS maps and profiles, Materialwissenschaft und Werkstoffechnik 48 (6), 485-494. https://doi.org/10.1002/mawe.201600737

Dietrich, D., G. Nolze, N. Del-Solar-Velarde, D. Nickel, T. Lampke, R. Chapoulie y L. J. Castillo

2018 The potential of EBSD and EDS for ceramics investigations - case studies on sherds of pre-Columbian pottery, Archaeometry 60(3), 489-501. https://doi.org/10.1111/arcm.12317

Disselhoff, $\mathrm{H}$.

1958 Cajamarca-Keramik von der Pampa von San José de Moro (Prov. Pacasmayo), Baessler-Archiv, Neue Folge 6, 181-193, Berlin.

Dollwetzel, P.

2012 Middle Horizon ceramic pigments. A meta-analysis of previous research on Peruvian ceramics in Bordeaux and Chemnitz, Reporte de investigación, Technische Universität, Chemnitz.

Donnan, C.

1972 Moche-Huari Murals from Northern Peru, Archaeology 25(2), 85-95.

Druc, I.

2011 Tradiciones alfareras del valle de Cajamarca y cuenca alta del Jequetepeque, Perú, Bulletin de l'Institut Francais d'Etudes Andines 40(2), 307-331. https://doi.org/10.4000/bifea.1438

2014 Alfares de Puémape: Un análisis petrográfico de cerámicas formativas de la costa norte de Perú, Arqueología y Sociedad 28, 275-288.

Druc, I., K. Inokuchi y Z. Shen

2013 Análisis de arcillas y material comparativo por medio de Difracción de rayos X y Petrografía para Kuntur Wasi, Cajamarca, Perú, Arqueología y Sociedad 26, 91-110.

Druc, I., S. Bertolino y A. Valley

2015 Rojo Grafitado is not graphite. A slow-science interpretation of the production of an Andean ceramic style, ponencia presentada al $80^{\text {th }}$ Annual Meeting of the Society of American Archaeology, San Francisco.

Nickel, D., L. J. Castillo, R. Chapoulie, N. Del-Solar-Velarde y P. Dollwetzel

2013 Material analysis of colored pre-Columbian ceramic shards: identification of black pigments on ceramics of the Huari Viñaque, Mochica Polícromo and Cajamarca Costeño styles, en: A. Hauptmann, O. Mecking, O y M. Prange (eds.), Archäometrie und Denkmalpflege 2013, Jahrestagung an der BauhausUniversität Weimar (25-28 September 2013), Dt. Bergbau-Museum Bochum, (Metalla. Sonderheft, 6), 148-153, Bochum.

Proulx, D.

2000 Nasca ceramic iconography: An overview, Studio Potter 29(1), 37-43.

Real Academia Española (RAE)

2018 Entrada: 'decoración'. Dirección web: http://dle.rae.es/srv/search?m=30\&w=decoraci\%C3\%B3n, visitado el 31 de diciembre de 2018.

Rohfritsch, A.

2006 Céramiques mochicas de la vallée de Jequetepeque (Pérou): étude technique et physico-chimique d'exemplaires provenant de Dos Cabezas et San José de Moro, mémoire de Master 2, Université Michelde-Montaigne Bordeaux 3, Burdeos.

2010 Contribución arqueométrica al estudio de las técnicas y de la organización de la producción de cerámica ritual en la sociedad mochica (150-850 d.C., costa norte del Perú), Bulletin de l'Institut Français d'Études Andines 39 (2), 389-412. https://doi.org/10.4000/bifea.2017

Sardos, N., N. Del-Solar-Velarde, R. Chapoulie y L. J. Castillo

2017 Caractérisation physico-chimique de pigments de peintures murales mochica: San José de Moro (VIIIe-Xe siècles apr. J.-C.), Journal de la société des américanistes 103 (1), 217-235. https://doi.org/10.4000/ jsa. 15017 


\section{Swann, C., S. Caspi y J. Carlson}

1999 Six stirrup handled Moche ceramic vessels from pre-Colombian Peru: a technical study applying PIXE spectrometry, Nuclear Instruments and Methods in Physics Research B 150, 571-575. https://doi. org/10.1016/S0168-583X(98)01062-3

Thiriet, C.

2008 Les Céramiques Cajamarca du Site de San Jose De Moro (Pérou), Étude comparative des groupes stylistiques «Serrano et Costeńo», mémoire de Master 2, Université Michel-de-Montaigne Bordeaux 3, Burdeos.

Tosi, J. A.

1960 Zonas de vida natural en el Perú: memoria explicativa sobre el mapa ecológico del Perú, Boletín 5, Instituto Interamericano de Ciencias Agrícolas de la OEA Zona Andina, Proyecto 39 Programa de Cooperación Técnica, Lima.

\section{Wright, V.}

2007 Étude de la polychromie des reliefs sur terre crue de la Huaca de la Luna, Trujillo, Pérou. tesis de doctorado en Prehistoria, Antropología y Etnología, Universidad Paris 1 Panthéon-Sorbonne, Paris.

Recibido: 31/01/2019

Aceptado: 31/07/2019 